\title{
Evaluation of peak-picking algorithms for protein mass spectrometry
}

Book or Report Section

Accepted Version

Bauer, C., Cramer, R. and Schuchhardt, J. (2011) Evaluation of peak-picking algorithms for protein mass spectrometry. In: Hamacher, M., Eisenacher, M. and Stephan, C. (eds.) Data mining in proteomics: from standards to applications. Methods in molecular biology, 696. Humana Press, USA, pp. 341-352. ISBN 9781607619864 doi: https://doi.org/10.1007/978-160761-987-1_22 Available at https://centaur.reading.ac.uk/18352/

It is advisable to refer to the publisher's version if you intend to cite from the work. See Guidance on citing.

To link to this article DOI: http://dx.doi.org/10.1007/978-1-60761-987-1_22

Publisher: Humana Press

Publisher statement: The original publication is available at www.springerlink.com

All outputs in CentAUR are protected by Intellectual Property Rights law, including copyright law. Copyright and IPR is retained by the creators or other copyright holders. Terms and conditions for use of this material are defined in the End User Agreement. 


\section{CentAUR}

Central Archive at the University of Reading

Reading's research outputs online 


\title{
Evaluation of peak picking algorithms for protein mass spectrometry
}

\author{
Chris Bauer, ${ }^{1, *}$, Rainer Cramer ${ }^{2}$, und Johannes Schuchhardt ${ }^{3}$ \\ ${ }^{1,3}$ MicroDiscovery GmbH, Berlin, Germany \\ ${ }^{2}$ The BioCentre and Department of Chemistry, The University of Reading, Whiteknights, \\ Reading, RG6 6AS, UK.
}

December 9, 2010

\begin{abstract}
Peak picking is an early key step in MS data analysis. We compare three commonly used approaches to peak picking and discuss their merits by means of statistical analysis. Methods investigated encompass signal to noise ratio (SNR), continuous wavelet transform (CWT) and a correlation-based approach using a gaussian template.

Functionality of the three methods is illustrated and discussed in a practical context using a mass spectral data set created with MALDI-TOF technology. Sensitivity and specificity are investigated using a manually defined reference set of peaks. As an additional criterion the robustness of the three methods is assessed by a perturbation analysis and illustrated using ROC curves.
\end{abstract}

Keywords: Peak picking, SNR, CWT, Gaussian template, Preprocessing, MALDITOF, ROC

\footnotetext{
${ }^{*}$ To whom correspondence should be addressed. Email: chris. bauer@microdicovery . com
} 


\section{Introduction}

Peak picking is an early key step in MS based proteomics and crucial for data analysis. It goes hand in hand with smoothing, baseline correction and peak alignment within a general workflow of preprocessing steps that allows for subsequent statistical data analysis and biological interpretation. Preprocessing of MS data aims at transforming a big amount of raw spectral data (usually $>30 \mathrm{~K}$ data points) into a much smaller, statistically manageable set of peaks. Subsequent data analysis will typically aim at biomarker discovery or sample classification. Comprising tens of thousands of data points in each spectrum, mass spectrometry data is inherently noisy. The main sources of noise are chemical in nature such as interference from matrix material and sample contamination or electrical noise which is dependent on the analytical set-up employed[1]. As a result, various algorithms differing in principle, implementation and performance have been proposed to address these problems.

A typical preprocessing workflow comprises the following three steps: (See figure 1 for a schematic illustration and exemplary visualization of each step)

- Data Smoothing: Smoothing mainly aims at removing high frequency noise. Beyond traditional signal processing techniques like Savitzky Golay filter [2], Mean/Median filter or Gaussian filters also wavelet based techniques are employed for data smoothing [3],[1].

- Baseline Correction: Baseline correction intends to remove low frequency noise and thus eliminates the correlation of nearby features. Typically methods like Top Hat filter[4], Loess derivative filters[5] or linear splines are applied to estimate the baseline.

- Peak Picking: The number of proposed methods for peak detection is immense. Most common algorithms make use of signal to noise ratio (SNR), continuous 


\section{Preprocessing}

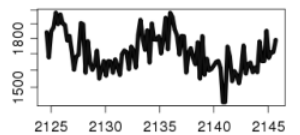

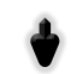

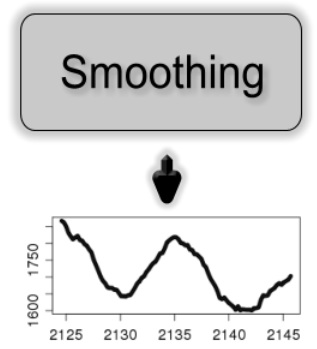

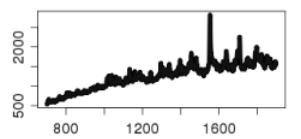

$\downarrow$

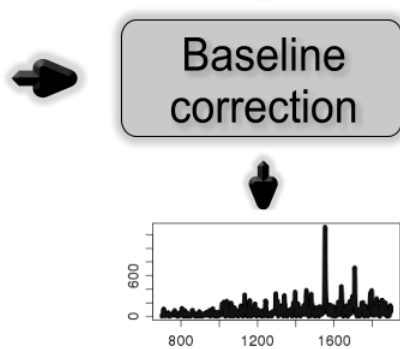

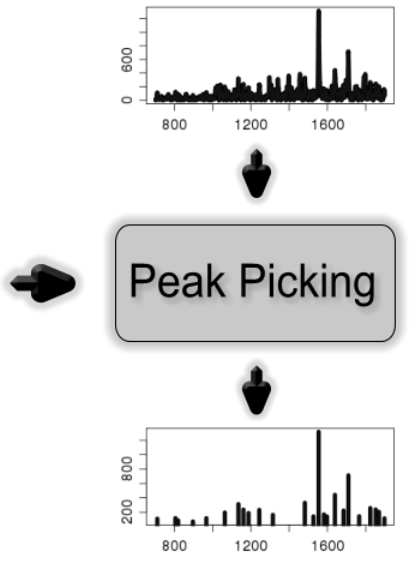

Figure 1: The preprocessing workflow is typically composed of the steps: smoothing, baseline correction and peak picking. In the course of preprocessing a raw spectrum is transformed into a peak list suitable for further statistical analysis.

wavelet transform (CWT) [6, 7] or model functions like Gaussian function used as templates for peak detection (see section 2 for more details).

A large variety of software packages implementing the complete workflow is available. Common software tools are R or Bioconductor packages msProcess or PROcess[8], Matlab packages LIMPIC[9] or Cromwell[3], the comprehensive C++ library OpenMS $[10],[11]$ and, of course, the proprietary software packages that come with the analytical equipment.

In this article we will focus on three different peak detection algorithms (SNR, CWT and Template-based approach), illustrate their principles in an intuitive manner and compare them in terms of sensitivity and specificity using ROC curves. We have selected these tree algorithms since they are very popular and widely-used. Furthermore all tree of them are very different and derived from distinctive theoretical motivations. Many extensions or combinations for these algorithms have emerged over the last years. For a more comprehensive overview including different techniques for smoothing and baseline correction see Yang et al. [12]. While Yang et al. give a 
comprehensive overview on publicly available software briefly describing the applied methods, our interest in this article is rather the demonstration of the working principle of the algorithms employed in these public software packages. Following up the evaluation of available peak detection algorithms by Yang et al. Liu et al. [13] compared different feature selection and classification algorithms in a similar way.

\subsection{Data set}

To evaluate the different algorithms we used data obtained by MALDI-TOF MS analysis of 259 blood plasma samples from 56 different mice taken at 5 different time points. Plasma MS profiles were obtained using an Ultraflex MALDI-TOF/TOF mass spectrometer (Bruker Daltonics, Bremen, Germany). Spectra were acquired automatically for the $\mathrm{m} / \mathrm{z}$ range of 700-10000. The amount of plasma obtained for each sample varied between 0 and $12 \mu \mathrm{l}$. Since $5 \mu \mathrm{l}$ were needed for each sample preparation, it was possible to perform up to two sample preparations. In a few cases only one or no sample preparation could be performed. From each sample preparation 4 replicate MALDI spectra were acquired, resulting in a total of up to 8 technical replicates per sample.

The total number of mass spectra acquired was more than 2100. Prior to any data processing described in this article technical replications are averaged reducing the number of spectra to 258 . For averaging multiple spectra we applied a peak alignment strategy[14]. 


\section{Peak Picking}

\subsection{Algorithms}

The three common peak detection algorithm we will focus on are Signal to noise ratio (SNR), Template based peak detection and Continuous Wavelet Transform (CWT). We have selected these three algorithms since they are very popular and widely-used. For the SNR and template-based approach we used an in-house implementation while for CWT we used the R package msProcess.

1. Signal to noise ratio (SNR): This is a very general approach. The essential part of this algorithm lies in the definition of noise. In statistics noise is often defined as variance or median absolute deviation (MAD) along different samples. In signal processing noise is often defined as the estimated background. For instance in the Bioconductor package PROcess[8] MAD of points within a window is used. For this analysis we follow the second approach defining noise as background of the spectrum. We estimated the background using Top Hat filter[4] with small window size. Having defined the noise we calculated the signal to noise ratio. Peaks are than identified by searching a local maximum of points withing a certain neighborhood (e.g. about expected peak width) having a SNR bigger than a given threshold.

2. Template based peak detection: This algorithm assumes that the peaks to be detected are shaped like some model function e.g. a gaussian function. With a running window the algorithm scans along the mass spectra and calculates the correlation (Pearson correlation coefficient) to a template gaussian function with predefined parameters. Thus the mass spectrum is transformed into a vector of correlation coefficients. Peak identification is done by searching for correlation values above a certain threshold. 
3. Continuous Wavelet Transform (CWT): CWT[6, 7] is a more sophisticated approach that is used to split the signal into different frequency ranges. Regarding the $\mathrm{m} / \mathrm{z}$ scale as generalized time scale, CWT constructs a time-frequency representation of the spectrum by mapping it from the time domain to the timescale domain. The essential part of CWT is the mother wavelet whose translated and scaled versions are used to generate daughter wavelets. The mother function we used for this evaluation is the second derivative of a gaussian function (Mexican Hat Wavelet). Peak picking typically includes the inspection of multiple scales. For peak detection (using R package msProcess) the peak candidate has to be clearly distinguishable from the background (parameter: snr.min) and visible across at least 7 scale domains (parameter: length.min) excluding the first three high frequency wavelet scales (parameter: scale.min). Excluding high frequency wavelets acts as filter for high frequency noise.

\subsection{Reference Peaks}

In order to evaluate the peak picking algorithms we defined a set of reference peaks. A peak picking algorithm can then be evaluated in terms of sensitivity (how many of the reference peaks are found) and specificity (how many of the found peaks are part of the reference set). An optimal algorithm has high sensitivity and high specificity.

The reference set was created in a semi-automatic process. To this end we initially picked peaks manually and subsequently optimized peak positions automatically. This procedure ensures a high quality reference set containing very prominent peaks as well as peaks situated in the rising or falling edge of another peak or peaks with poor signal intensities. All in all the reference set contained a total of 381 peaks. 


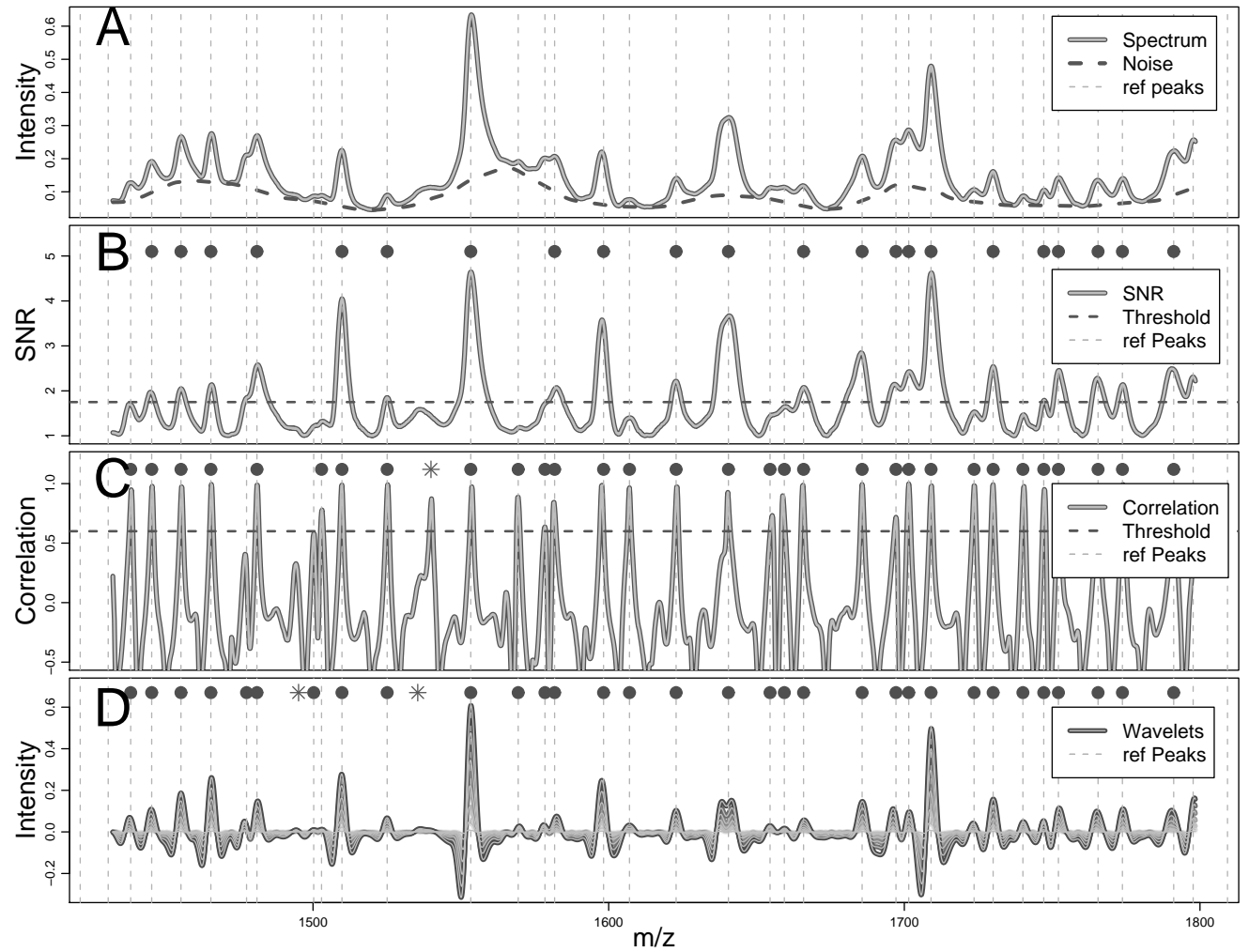

Figure 2: Comparison of the 3 different peak picking algorithms for the $\mathrm{m} / \mathrm{z}$-range 1400 - 1800. A: mean spectrum and noise/background (dashed line); B: SNR and threshold used for peak picking (horizontal dashed line); C: correlation coefficient and threshold (horizontal dashed line); D: first 7 wavelets. The vertical dashed lines are reference peaks. Marks above the plot indicate identified peaks ( $d o t=$ contained in the reference set; asterisk $=$ not in reference set - false positives) 


\subsection{Comparing peak picking algorithms}

Figure 2 gives a graphical impression of how the different algorithms are working. The first box shows the mean intensity spectrum of the complete data set in a mass window of 1400-1800 Da. The noise level was defined as baseline calculated using Top Hat filter (see dashed line). The 33 peaks from the reference set within this mass window (see section 2.2) are indicated as vertical dashed lines.

The second part of figure 2 shows the signal to noise ratio along the mass window of the mean spectrum. The SNR threshold used for peak identification was 1.75 indicated as horizontal dashed line. Using SNR we identified 22 peaks in this mass range whereas we found $69 \%$ of our reference peaks (with the SNR threshold of 1.75). With this threshold we did not find any peak that was not part of the reference set.

The third box in figure 2 visualizes the performance of template-based peak detection. The correlation coefficients along the spectrum are shown. The correlation threshold of 0.6 is indicated as horizontal dashed line. All in all we found 31 of the 33 reference peaks (94\%) indicated as dots above the peaks. We also found one peak that is not within the reference set (false positive) shown as asterisk above the peak.

The last part of figure 2 demonstrates the peak picking using wavelet transform. The first 7 daughter wavelets are shown. Compared to the other two methods the peak picking is complicated by the fact that information from different time-scale domains has to be combined (see chapter 2.1 for more details). The reference peaks again appear as vertical dashed lines and the picked peaks are marked above the peaks. Using CWT we identified $97 \%$ of the peaks but also got two false positive hits (marked with asterisks above the peaks). 


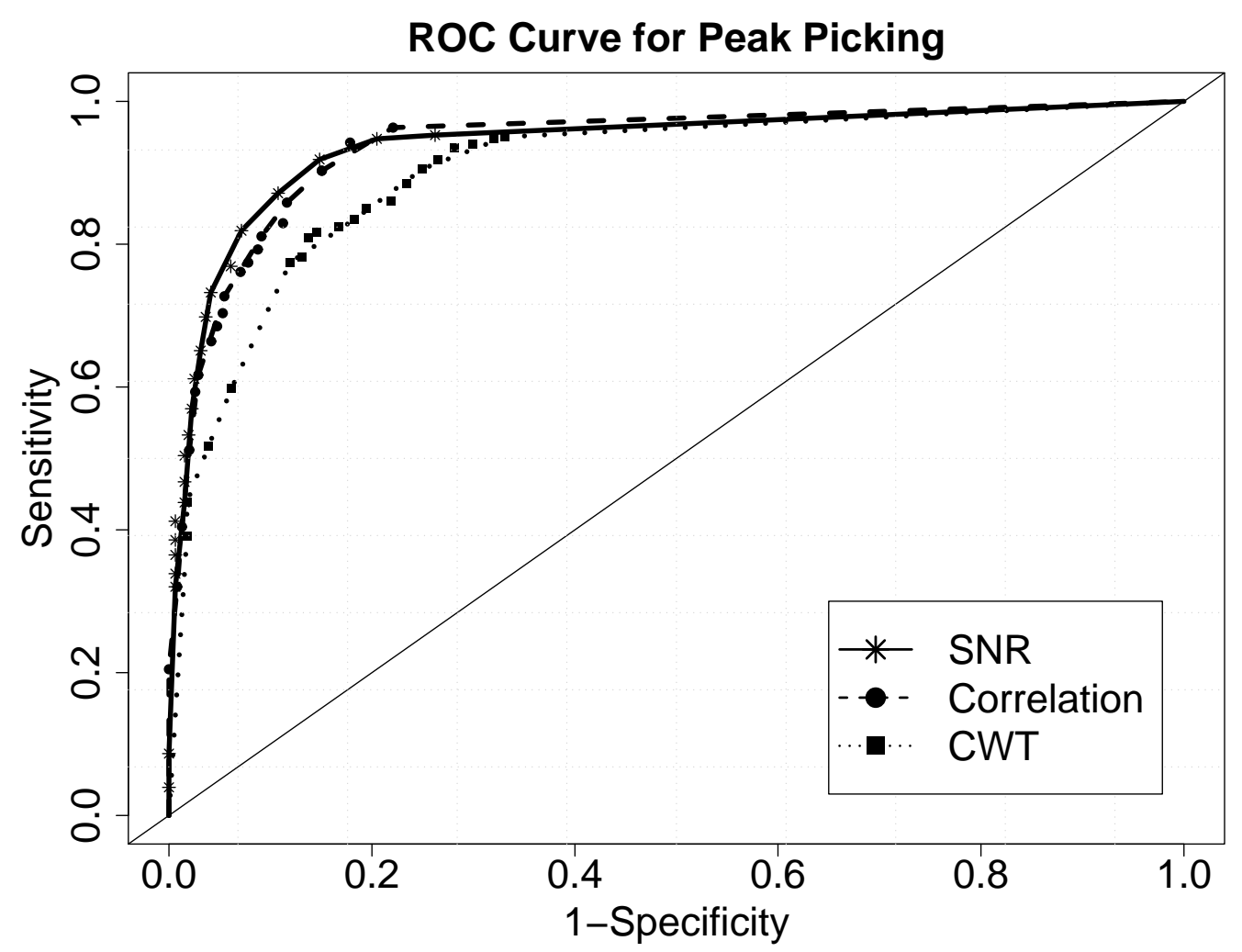

Figure 3: ROC curves presenting the performance of the three different peak picking algorithms: SNR (full line), Correlations with gaussian function (dashed line) and Continuous Wavelet Transform (pointed line). The ROC curves are calculated by scanning the threshold values of the different algorithms.

\subsection{Evaluating peak picking algorithms}

As already mentioned in section 2.2 the reference peak set can be used to calculate values for sensitivity and specificity. That in turn can be used to generate ROC curves (see figure 3). ROC curves are calculated by scanning the threshold values of the different algorithms e.g. changing the correlation threshold in the template based approach (for an illustration of the threshold operation see figure 2).

\subsubsection{Stability}

The combination of smoothing and baseline correction defines a bandpassfilter removing high and low frequency noise. Parameter tuning of those preprocessing steps 

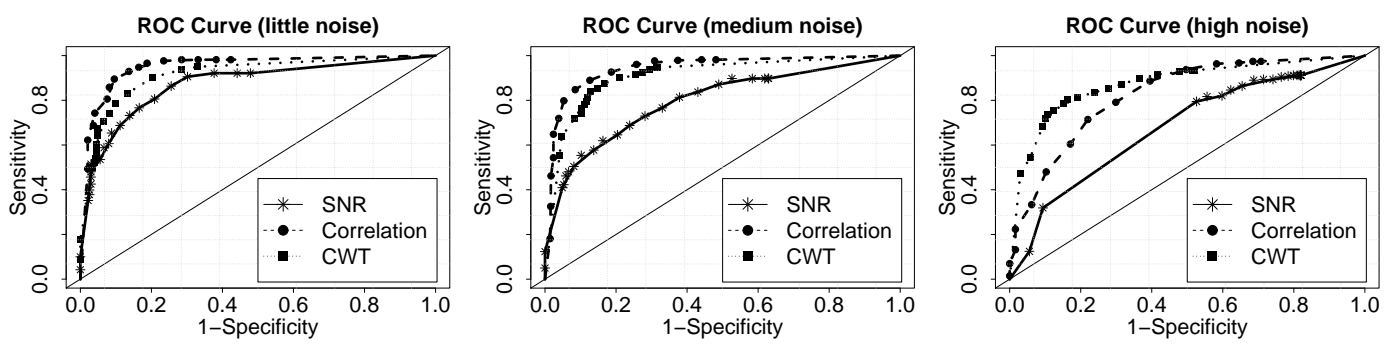

Figure 4: ROC curves for the three different peak picking algorithm on noisy Data: SNR (full lines), Correlations with gaussian function (dashed lines) and Continuous Wavelet Transform (pointed lines).

affects the signal and noise the peak picking algorithms have to deal with. In order to evaluate the sensitivity to noise we added different quantities of high frequency noise (white noise). Since the observed error behavior for MS spectra indicates a multiplicative error behavior on log scale data (not shown), we added a normally distributed noise with mean $=0$ and an error of $2 \%, 4 \%$ and $10 \%$. The performances of the three methods are affected to a very different degree (see figure 4 for the ROC curves). The signal to noise ratio is very sensitive to noise and the ROC curve worsens dramatically. The other two algorithms are much more robust. While on perfectly smoothed data the template correlation approach seems to be the method of choice, for noisy data the advantage of the template-based approach decreases and CWT shows the best performance. In conclusion the three presented peak picking algorithms show a different sensitivity to noise and therefore to the number of spectra and the choice of parameters for preprocessing steps.

In order to get more insight into how noise influences the peak detection figure 5 gives a demonstration of the algorithm's performances on noisy data. The first row shows the raw spectra and the baseline. Signal to noise ratio is depicted in the second row of figure 5. Here the noise is even amplified due to the ratio calculation leading to an increased number of false positive peaks (see the asterisks). The template correlation approach is more robust but since this method is only assessing the shape and not the intensity even small fluctuations may result in high correlation coefficients. Thus 


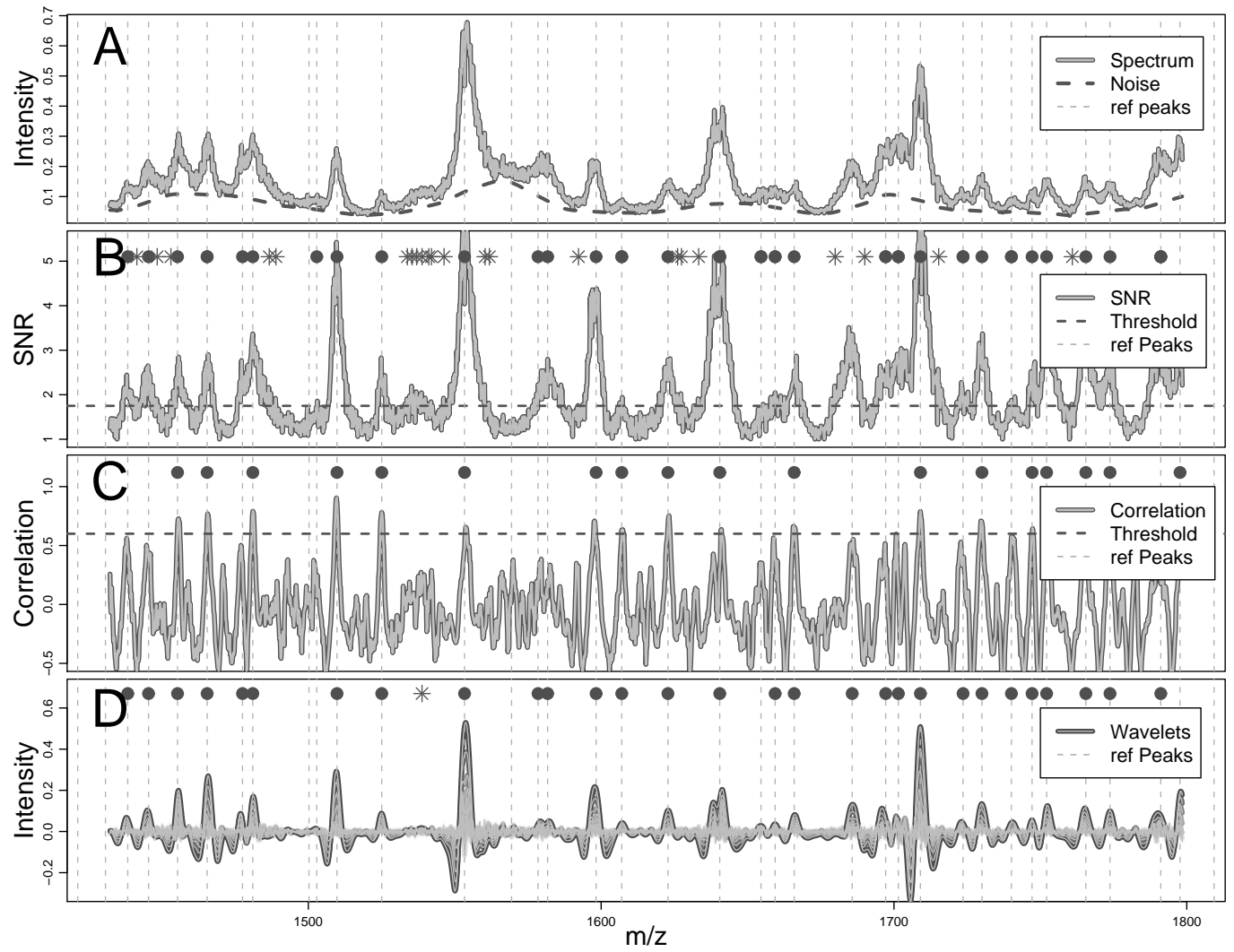

Figure 5: Comparison of the 3 different peak picking algorithms on noisy data for the $\mathrm{m} / \mathrm{z}$ range of $1400-1800$. High frequency noise was added to the spectrum as described in the text. A: mean spectrum and background (dashed line); B: SNR and threshold used for peak picking (horizontal dashed line); C: correlation coefficient and threshold (horizontal dashed line); D: first 7 wavelets. The vertical dashed lines are reference peaks. Marks above the plot indicate peaks identified with the different algorithms (dot $=$ contained in the reference set; asterisk $=$ not in the reference set).

peaks are not clearly distinguishable from background noise any more. For noisy data CWT outperforms the other methods since CWT intrinsically acts like a smoothing filter on the data. Even if the first wavelets are noisy, the lower frequency scales are very smooth (see lower row of figure 5). Hence high frequency noise does not affect the algorithm's performance strongly as high frequency wavelets that include most of the noise are filtered out. 


\section{Discussion}

The three different peak picking algorithms investigated here are distinct in terms of complexity, performance and stability. But all three methods have a common parameter: the estimated peak width. There are different ways to estimate the optimal peak width. For instance OpenMS [10],[11] as a freely available MS processing library offers the possibility to measure the peak width manually using graphical interface or the peak width can be estimated by the CWT algorithm itself.

For an overview of the advantages and disadvantages of the algorithms see table 1. Signal to noise ratio as a universally used signal processing technique is computationally fast, easy to implement and shows good performance on smoothed data. However, it is not very specific for this task as it ignores the shape of the peak. Since the noise is an integral part of the algorithm it is very sensitive to noise and therefore strongly depends on the quality of the data and on the performance of previously performed smoothing and baseline correction steps.

The template-based approach is much more specific for the peak picking task assuming peaks to be shaped like a gaussian function. This assumption, however, might often not be exactly applicable because peaks may show a considerable asymmetry. Depending on the experimental parameters, particularly laser energy, significant deviation from a Gaussian peak shape can be globally obtained. Although this method has only a few parameters, it appears rather robust for lower levels of noise. However for high levels of noise the performance decreases.

CWT is like SNR a very universal signal processing technique used for many different tasks. Contrary to SNR the algorithm is complex and is computationally expensive. The large number of parameters allows for tuning CWT to be very specific for this task taking into account the shape of the peak. As smoothing is an intrinsic part of the algorithm CWT is very robust even for substantial amounts of noise. On the other 


\begin{tabular}{|c|c|c|}
\hline Method & PRO & CONTRA \\
\hline SNR & $\begin{array}{l}\text { - } \text { simple - easy to implement } \\
\text { - fast performance } \\
\text { - only few parameters }\end{array}$ & $\begin{array}{l}\text { - depends on the definition of } \\
\text { noise } \\
\text { - unstable - very sensitive to } \\
\text { noise } \\
\text { - ignoring peak shape }\end{array}$ \\
\hline $\begin{array}{l}\text { Template } \\
\text { Correlation }\end{array}$ & $\begin{array}{l}\text { - } \text { simple - easy to implement } \\
\text { - only few parameters } \\
\text { - stable for small noise }\end{array}$ & $\begin{array}{l}\text { - detection favors gaussian } \\
\text { shaped peaks } \\
\text { - sensitive to high noise }\end{array}$ \\
\hline CWT & $\begin{array}{l}\text { - stable even for massive noise } \\
\text { - internal data smoothing } \\
\text { - flexible - tuneable }\end{array}$ & $\begin{array}{l}\text { - complicated algorithm } \\
\text { - slow performance } \\
\text { - difficult to tune - high number } \\
\text { of parameters }\end{array}$ \\
\hline
\end{tabular}

Table 1: Summary of advantages and disadvantages of the three presented peak picking algorithms.

hand tuning of the algorithm is difficult due to the large set of parameters.

For perfectly smoothed data all three methods show good performances but CWT seems to be little worse than the other two. For data including a substantial amount of noise CWT clearly outperforms the other methods in terms of sensitivity and specificity.

Both, the template based approach and CWT show good performances including a robustness for noise. Figure 6 shows two example peaks for the different peak detection using these two algorithms. In the upper row the peak at m/z 1846 was identified only with CWT while in the lower row the peak at m/z 4052 was detected only with the template-based method. The shortcoming of the template-based approach is clearly visible since the peak at m/z 1846 is not shaped like a gaussian function resulting in lower correlation coefficients. Hence this peak could not be detected using a gaussian function as template. In contrast the peak at m/z 4052 shows a good matching gaussian shape, facilitating peak detection by correlation. CWT does not find this peak since there are not enough wavelets above threshold (in this case there are only 

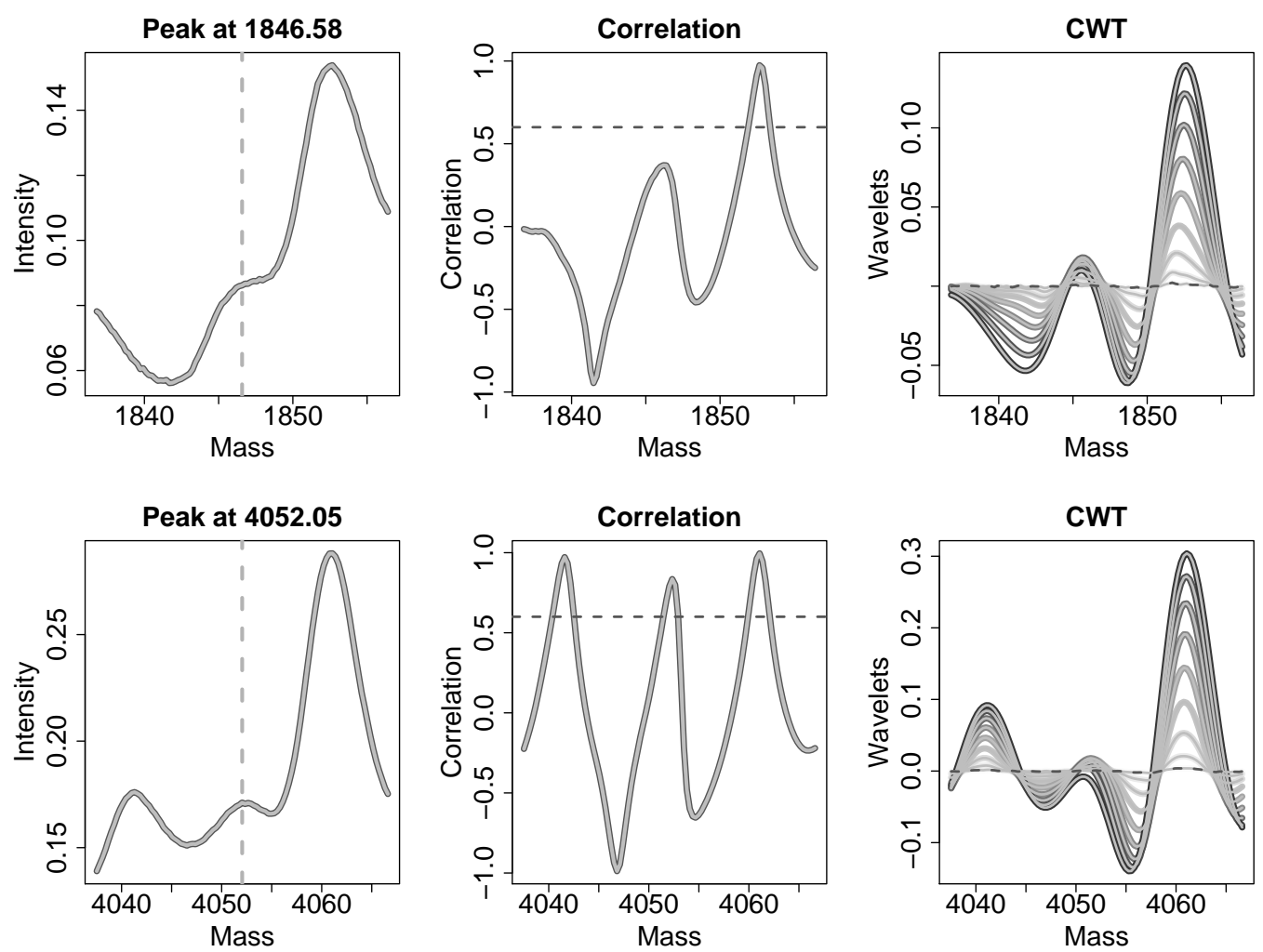

Figure 6: Peaks found with CWT and not with template based approach (upper part) and vice versa (lower part), first column: spectrum, second column: correlation coefficient and correlation threshold, third column: first 9 wavelets and noise (dashed line).

5 wavelets above the noise level but the algorithm requires at least 7).

The reference set we used for evaluation was manually created assuming the human eye to be a good peak detector. With this procedure we assure that the reference set is constructed without giving preference to any algorithm. Looking at the spectra and the visualization of the three algorithms (figure 2) we see that there is one peak identified with correlation based approach and CWT (indicated as asterisks) and even with a higher SNR that was not classified as a peak using the human eye. However, remarkably the three algorithms differ in exactly this peak underlining that in general peak picking is a non-trivial task. 


\section{Notes:}

$\rightarrow$ For MALDI-TOF data adequate preprocessing is required in order to allow subsequent statistical data analysis such as biomarker discovery or sample classification.

$\rightarrow$ Preprocessing workflow typically comprises algorithms for data smoothing such as Mean filter or Savitzki Goley filter, baseline correction like Top Hat filter or Loess derived filters and peak picking such as SNR, CWT or template based approaches.

$\rightarrow$ The main objective is to transform the big amount of raw spectral data into a much smaller, statistically manageable set of peaks.

$\rightarrow$ The number of algorithms implementing peak picking is large. The various algorithms differ in performance, implementation and theoretical motivation.

$\rightarrow$ Various common software tools are available designed to address the preprocessing workflow. They are based on different platforms including $\mathrm{R}$ and Matlab packages as well as stand-alone $\mathrm{C}++$ applications.

$\rightarrow$ Approaches based on SNR are rather simple, easy to use and fast but also sensitive for noise. Moreover the shape of the peak is ignored completely.

$\rightarrow$ Template-based approaches are simple, easy to use and robust to limited noise. But they can only detect peaks shaped like the used template function and they are vulnerable to strong noise.

$\rightarrow$ CWT shows good performances and is stable even for strong noise but more complicated, difficult to tune and therefore harder to use and understand.

$\rightarrow$ Every algorithm has pros and cons as it fails in finding certain types of peaks. 
Template based approach fails to detect peaks differing in shape from used template. CWT tends to miss thin peaks surrounded by higher ones.

$\rightarrow$ The definition of the reference peak set is a crucial step for evaluating the different algorithms. Neither the human eye nor some automatic peak detection algorithm can guarantee to detect all peaks. Still, regarding a sensitivity and specificity of 0.9 , the majority of the peak show good agreement of the used algorithms and the human eye.

\section{References}

[1] D. Kwon, M. Vannucci, J.J. Song, J. Jeong, and R.M. Pfeiffer. A novel waveletbased thresholding method for the pre-processing of mass spectrometry data that accounts for heterogeneous noise. Proteomics, 8:3019-3029, Aug 2008.

[2] Abraham Savitzky and Marcel J.E. Golay. Smoothing and differentiation of data by simplified least squares procedures. Analytical Chemistry, 36(8):1627-1639, 1964.

[3] K.R. Coombes, S. Tsavachidis, J.S. Morris, K.A. Baggerly, M.C. Hung, and H.M. Kuerer. Improved peak detection and quantification of mass spectrometry data acquired from surface-enhanced laser desorption and ionization by denoising spectra with the undecimated discrete wavelet transform. Proteomics, 5:4107-4117, Nov 2005.

[4] Anne C Sauve and Terence P. Speed. Normalization, baseline correction and alignment of high-throughput mass spectrometry data. 2004.

[5] W.S. Cleveland, E. Grosse, and W.M. Shyu. Local regression models, pages pp. 309-376. Wadsworth, 1992. 
[6] E. Lange, C. Gröpl, K. Reinert, O. Kohlbacher, and A. Hildebrandt. Highaccuracy peak picking of proteomics data using wavelet techniques. Pac Symp Biocomput, pages 243-254, 2006.

[7] P. Du, W. A. Kibbe, and S. M. Lin. Improved peak detection in mass spectrum by incorporating continuous wavelet transform-based pattern matching. Bioinformatics, 22:2059-2065, Sep 2006.

[8] Robert Gentleman and Vince Carey and Wolfgang Huber and Rafael Irizarry and Sandrine Dudoit, editor. Bioinformatics and Computational Biology Solutions Using $R$ and Bioconductor. Springer Verlag, 2005.

[9] D. Mantini, F. Petrucci, D. Pieragostino, P. Del Boccio, M. Di Nicola, C. Di Ilio, G. Federici, P. Sacchetta, S. Comani, and A. Urbani. LIMPIC: a computational method for the separation of protein MALDI-TOF-MS signals from noise. BMC Bioinformatics, 8:101, 2007.

[10] O. Kohlbacher, K. Reinert, C. Gröpl, E. Lange, N. Pfeifer, O. Schulz-Trieglaff, and M. Sturm. TOPP-the OpenMS proteomics pipeline. Bioinformatics, 23:e191-197, Jan 2007.

[11] M. Sturm, A. Bertsch, C. Gröpl, A. Hildebrandt, R. Hussong, E. Lange, N. Pfeifer, O. Schulz-Trieglaff, A. Zerck, K. Reinert, and O. Kohlbacher. OpenMS - an open-source software framework for mass spectrometry. $B M C$ Bioinformatics, 9:163, 2008.

[12] C. Yang, Z. He, and W. Yu. Comparison of public peak detection algorithms for MALDI mass spectrometry data analysis. BMC Bioinformatics, 10:4, 2009.

[13] Q. Liu, A. H. Sung, M. Qiao, Z. Chen, J. Y. Yang, M. Q. Yang, X. Huang, and Y. Deng. Comparison of feature selection and classification for MALDI-MS data. BMC Genomics, 10 Suppl 1:S3, 2009. 
[14] N. Jeffries. Algorithms for alignment of mass spectrometry proteomic data. Bioinformatics, 21:3066-3073, Jul 2005. 